\title{
A Parametric Study of an Acoustic Black Hole on a Beam
}

\author{
K. Hook, ${ }^{1}$ J. Cheer, ${ }^{1,}$ a) and S. Daley ${ }^{1}$ \\ Institute of Sound and Vibration Research, The University of Southampton, \\ Southampton, SO17 1BJ, United Kingdom
}

(Dated: 29 April 2019) 
Acoustic black holes (ABHs) are geometric structural features that provide a potential lightweight damping solution for flexural vibrations. In this article, a parametric study of an $\mathrm{ABH}$ on a beam has been carried out to assess how practical design constraints affect its behaviour, thus providing detailed insight into design trade-offs. The reflection coefficient of the ABH has been calculated for each taper profile, parameterised via the tip-height, taper-length and power-law and it has been shown to exhibit spectral bands of low reflection. These bands have been related to the modes of the ABH cell and become more closely spaced in frequency as the ABH parameters are suitably varied. This suggests that ABH design should maximise the modal density to minimise the broadband reflection coefficient, however, the minimum level of reflection is also dependent on the power-law and tip-height. Consequently, broadband reflection values have been used to show that optimum power-law and tip-height settings exist that achieve a balance between maximum modal density and minimum level of reflection. Additionally, at discrete frequencies, in cases where tip-height and taper-length are practically constrained, the power law can be tuned to maximise performance. Finally, an experimental study is used to validate the results.

a)j.cheer@soton.ac.uk 


\section{INTRODUCTION}

There is a requirement for lightweight vibration control solutions in a variety of application areas. One such solution is the so-called 'acoustic black hole' (ABH), which relies on introducing modifications to the design of the structure that reduce the structural wave speed and thus allow their control. The desired design modifications are generally achieved via a change in the geometry of the structure ${ }^{1}$. Specifically, when the edge of a beam or plate is tapered, over a distance comparable to or larger than the flexural wavelength ${ }^{2}$, the flexural wave speed decreases as the beam or plate thickness decreases. This phenomenon was described by Mironov in $1988^{3}$ and later termed the 'acoustic black hole' effect by Krylov and Tilman ${ }^{1}$. Figure 1 shows a simple example of an $\mathrm{ABH}$, where a beam has been tapered to a point via a power law curve. As flexural waves propagate towards the tip, the wave speed is reduced following the relationship

$$
c_{f}(x)=\left(\frac{E h^{2}(x)}{12 \rho_{s}}\right)^{\frac{1}{4}} \omega^{\frac{1}{2}},
$$

where $E$ is the Young's modulus of the beam material, $\rho_{s}$ is the volume density of the beam material and $h(x)$ is the height function. Equation 1 shows that the wave speed in a tapered beam is proportional to the square root of the beam height and, therefore, the wave speed will decrease along the length of a taper with a decreasing height.

From equation 1 it can be deduced that, theoretically, if the taper reduces to zero thickness, then the velocity of the propagating wave will converge to zero. In this idealised case, the propagating wave will never reach the tip of the taper and will, therefore, not be reflected from the end of the tapered beam; hence the analogy to a black hole. In reality, the tapered 


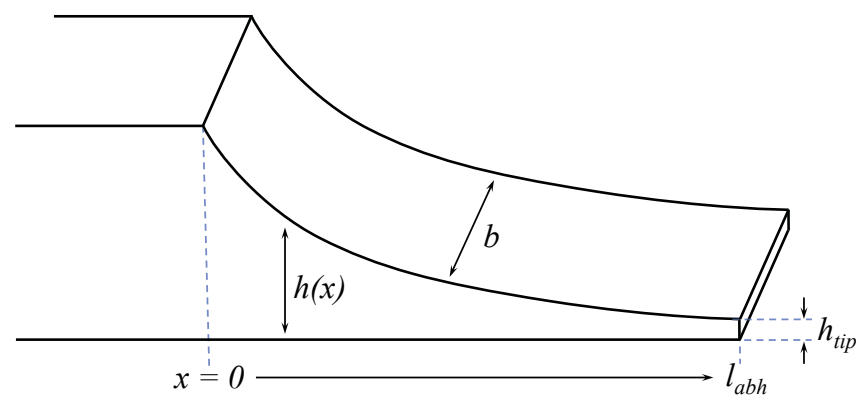

FIG. 1. A diagram of an ABH taper with characteristic features indicated as: width, $b$; tip height, $h_{\text {tip }}$; taper length, $l_{a b h}$ and height function, $h(x)$.

section will have a finite length and the tip height will be limited by both manufacturing and structural integrity limitations ${ }^{4-6}$. As a result of these practical limits, it has previously been shown that the $\mathrm{ABH}$ effect is negligible for practical tip heights, but that significant vibration reduction can be achieved through the addition of a thin layer of passive damping material $^{1}$.

The significant effect of a thin damping layer on the vibration control provided by a practical $\mathrm{ABH}$ can be understood by considering the change in the flexural wavelength, $\lambda_{f}$, along the length of the taper, which can be expressed as

$$
\lambda_{f}(x)=\left(\frac{E h^{2}(x)}{12 \rho_{s}}\right)^{\frac{1}{4}}\left(\frac{2 \pi}{f_{0}}\right)^{\frac{1}{2}}
$$

where $f_{0}$ is the frequency of the wave. Equation 2 shows that as a wave travels down the taper, its wavelength decreases. It is well known that shorter wavelength vibrations are more easily attenuated using traditional passive damping treatments and this, therefore, explains why adding a thin damping layer to a practical ABH provides a significant level of performance. This effect has been practically demonstrated for thin viscoelastic damping layers added to either one or both sides of the $\operatorname{taper}^{1,7,8}$ and composite plates ${ }^{9}$. Additionally, 
it has previously been shown that the whole taper need not be covered by the damping layer ${ }^{10}$ and that the thickness of the damping layer can be up to six times the thickness of the base layer before the increase in loss factor diminishes ${ }^{11-13}$.

In addition to practical ABHs requiring an additional damping layer to be applied to the taper, previous research has also investigated how the geometrical properties of the taper should be defined for optimal performance. In particular, it has been shown that the taper profile, tip height and taper length influence the performance of the $\mathrm{ABH}^{1}$. Although the literature has broadly shown that maximising the taper length and minimising the tip height will maximise the performance, it has more recently been demonstrated that the selection of the power law profile requires a tradeoff ${ }^{14}$. That is, a high power law is predicted from the original theoretical analysis ${ }^{1,3}$ to maximise the reduction in the wave speed within the taper and thus minimise the reflection coefficient, however, a high power law simultaneously violates the smoothness criterion inherent in the analysis utilised in the original modelling approach $^{14}$. As a result, Shepherd et al proposed a method of selecting the power law profile to reach an optimal tradeoff ${ }^{15}$. Since then, further physical insight into this tradeoff has been gained through more complete models, which include both the uniform and tapered sections of the structure ${ }^{12,16-20}$. Within this body of work it has been shown that high power laws, which were penalised in ${ }^{15}$ due to violation of the smoothness criterion, physically result in significant reflection from the junction between the uniform section of the beam and the taper due to the rapid impedance change at this point ${ }^{18,21}$.

Although significant physical insight has already been demonstrated through various studies of ABHs, these previous investigations have generally focused on a specific design 
parameter ${ }^{12,19}$, or considered only a small number of design cases over frequency or a parameter sweep at a specific frequency ${ }^{1,8,16,17,20}$. Therefore, to provide more detailed insight in to the effect that the geometrical design parameters have on the performance of an $\mathrm{ABH}$ terminating a beam, this paper presents a full parametric design study, which considers the influence of the tip height, taper length and power law on the overall reflection coefficient over a broad frequency range. This study provides new physical insight into the design of an $\mathrm{ABH}$ and is complemented by a corresponding modal analysis. This modal analysis builds on previous work that demonstrates the link between the ABH performance and the modal density ${ }^{17}$ and the link between the bands of low reflection and the local modes of the $\mathrm{ABH}$ cell ${ }^{22}$, by highlighting in detail how the local modes of the ABH are influenced by the geometrical design parameters. Furthermore, using the data from the full parametric design sweep, an investigation is also presented here into how the geometrical parameters should be selected for optimal broadband performance, which is particularly useful when selecting the optimum power law for an $\mathrm{ABH}$ design with practical constraints on the taper length and tip height, but also shows new insight into the selection of the tip-height.

The presented investigation is laid out in the following structure. In section II, a numerical model of a lightly damped ABH taper on a beam is presented. This is followed, in Section III, by the full parametric study, which investigates the effect that varying each geometrical parameter has on the reflection coefficient of the $\mathrm{ABH}$ on a beam over frequency and how this can be related to the modes of the $\mathrm{ABH}$. An investigation into the optimal broadband performance is then presented in Section IIID. In section IV, an experimental case study is presented, which serves to validate how the characteristics of the reflection coefficient can 
be related to the modes of the ABH cell as indicated by the numerical study. Finally, in Section $\mathrm{V}$, the conclusions of this investigation are presented.

\section{NUMERICAL MODELLING}

In order to carry out the parametric study, a numerical model of an $\mathrm{ABH}$ on the end of a beam has been developed. The numerical model was implemented using the finite element modelling software COMSOL Multiphysics, with the beam physics module using Timoshenko beam theory. This allows the beam and taper to be defined as a $2 \mathrm{D}$ crosssection, before being assigned a finite width. Although this 1-dimensional model neglects torsional modes and flexural modes across the width of the beam, it allows a comprehensive parametric study to be carried out within practical computational limitations. The use of 1-dimensional models in the study of ABHs is common and has previously been successfully utilised in a number of studies such $\mathrm{as}^{21,22}$. This section will describe the geometry and physical properties of the modelled beam, discuss the meshing procedure and outline how the generated data will be processed to obtain the reflection coefficient.

\section{A. Model Geometry}

A diagram of the model geometry is shown in Figure 2 and the range of parameters used in the following parametric study are detailed in Table I. In the following parametric study, the uniform beam geometry is kept constant, with the beam height, width and length as defined in Table I. The ABH geometrical properties (excluding its width) are, however, varied over practical ranges, as also defined in Table I. Although a variety of taper profiles 
Parameter

Beam height

Beam length

Beam/ABH width

$\mathrm{ABH}$ tip height

$\mathrm{ABH}$ taper length

$\mathrm{ABH}$ power law

Excitation force
Symbol

$h(0)$

$l_{\text {beam }}$

$b$

$h_{\text {tip }}$

$l_{a b h}$

$\mu$

$\mathbf{F}$

TABLE I. The parameters used in the model geometry.

\begin{tabular}{ccc}
\hline \hline Parameter & Symbol & Value / Range of Values \\
\hline Beam height & $h(0)$ & $10 \mathrm{~mm}$ \\
Beam length & $l_{\text {beam }}$ & $300 \mathrm{~mm}$ \\
Beam/ABH width & $b$ & $40 \mathrm{~mm}$ \\
ABH tip height & $h_{\text {tip }}$ & $0.01 \mathrm{~mm}-3 \mathrm{~mm}$ \\
ABH taper length & $l_{a b h}$ & $10 \mathrm{~mm}-300 \mathrm{~mm}$ \\
ABH power law & $\mu$ & $1-10$ \\
Excitation force & $\mathbf{F}$ & $1 \mathrm{~N}$ \\
\hline \hline
\end{tabular}

123

have been investigated in the literature ${ }^{1,18}$, the differences in performance are relatively

$$
h(x)=\varepsilon x^{\mu}+h_{t i p},
$$

where $\varepsilon=h(0)-h_{\text {tip }}$ is a scaling factor, $x$ is the position along the taper, $\mu$ is the power law of the taper that defines the gradient and $h_{t i p}$ is the tip height at the end of the taper.

Figure 2 also shows the position of two sensors on the beam and a point force excitation, which was symetrically located so as to only excite longitudinal flexural motion. The sensors were positioned midway along the beam section, separated by $\Delta_{x}=2 \mathrm{~cm}$, to allow extraction 


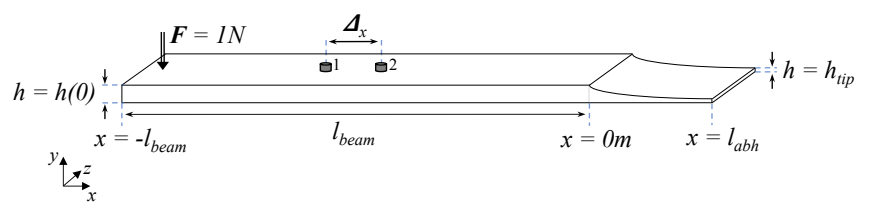

FIG. 2. A diagram of the modelled beam and ABH (not to scale). The locations of the force excitation and sensing locations are also indicated.

of the reflection coefficient, as described in Section II C. The length of the uniform beam was chosen to be sufficient such that evanescent components could be neglected in the analysis, as also discussed in Section II C. The beam and ABH were both assumed to be constructed from aluminium alloy 6082-T6 and some inherent damping has been implemented as an isotropic loss with a factor of $\eta_{\text {Beam }}=0.0001$, which is consistent with other studies ${ }^{11,16,23}$. To model the additional damping required in the practical $\mathrm{ABH}$, further damping was included in the tapered section of the beam by applying an isotropic loss factor of $\eta_{A B H}=0.2$ and the additional mass of this damping layer was modelled by an evenly distributed mass of $11.9 \mathrm{~g}$ along the length of the taper. This level of damping and additional mass were calculated to match the damping layer used in the experimental implementation discussed in Section IV. The initial conditions of the $\mathrm{ABH}$ were set to stationary and the boundary conditions of all the edges were set to free.

\section{B. Meshing}

Typically, a minimum of 6 elements should be used per wavelength when constructing a finite element model ${ }^{24}$. However, as noted in the introduction, the wavelength varies in the tapered section and, therefore, some care must be paid to the meshing of the ABH. To 
validate the meshing requirement for the $\mathrm{ABH}$, a convergence study was performed with the number of (edge) elements per wavelength ranging from $n=1$ to $n=20$. To ensure a sufficiently fine mesh within the $\mathrm{ABH}$, the reference wavelength was taken from the tip of the $\mathrm{ABH}$, where the wavelength is the shortest, ensuring that there is a minimum of $n$ elements per wavelength at all points along the taper. Although the resolution of the mesh could be varied over the length of the taper to improve efficiency, this is not straightforward because the details of the variation in the wavelength depend on the specific properties of the $\mathrm{ABH}$, which will be investigated in Section III. Therefore, although reducing the computational efficiency, selecting the mesh according to the minimum wavelength ensures sufficient accuracy at all points. To ensure that the meshing procedure is sufficient for the full parameter sweep, a mesh convergence study has been conducted for the longest taper $(30 \mathrm{~cm})$, smallest tip height $(0.01 \mathrm{~mm})$ and a power law of $\mu=10$, which gives the largest variation in the wavelength along the taper. To assess the convergence, the mean of the magnitude of the displacements measured at each element was calculated at the upper frequency of interest $(10 \mathrm{kHz})$, using an increasing number of elements per wavelength and the results are shown in Figure 3. From these results it can be seen that the mean of the magnitude of the displacement per element has converged to a constant value when there are approximately 10 or more elements per wavelength and, therefore, this value has been used in the following parametric study.

Based on the results of the convergence study, Figure 4 shows how the total number of elements required to model the taper section varies with the tip height at the maximum frequency of interest in the following study, which is $10 \mathrm{kHz}$. From this plot it can be 


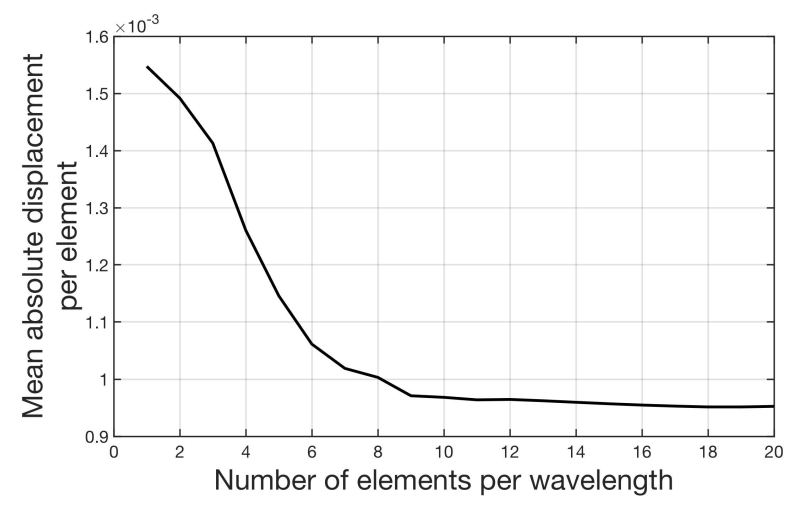

FIG. 3. The mean of the magnitude of the displacement per element plotted against the number of elements per wavelength at $10 \mathrm{kHz}$.

seen that the number of elements required rapidly decreases as the tip height increases. A convergence study was also carried out to ensure that a sufficient number of elements were used to model the uniform beam section, which is constant over the various parameterisations and a total of 10 elements per wavelength were used, giving a total of 32 elements in the beam section at the upper frequency of interest. The change in the size of the mesh elements between the $\mathrm{ABH}$ and the beam section, which is related to the difference in the minimum wavelength in each section, is depicted in Figure 5.

\section{Wave decomposition in a beam}

Wave decomposition is the separation of a measured disturbance into the individual wave components. For example, wave decomposition has previously been used to calculate the wave components in both acoustic systems ${ }^{25}$ and in structures such as beams ${ }^{26,27}$ and $\mathrm{ABHs}^{28}$. To investigate the performance of the $\mathrm{ABH}$, a wave decomposition, based on ${ }^{28}$, will 


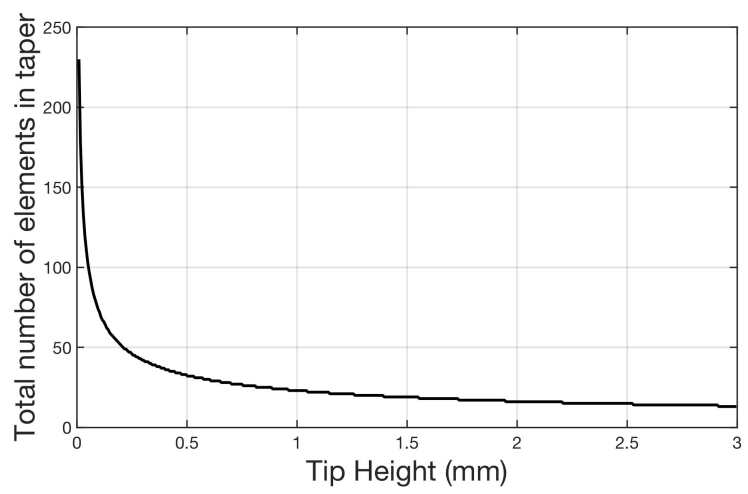

FIG. 4. The number of elements required to model a $7 \mathrm{~cm}$ taper with a power law of 6 for each tip height increasing from $0.01 \mathrm{~mm}$ to $3 \mathrm{~mm}$ with 10 elements per wavelength at a $10 \mathrm{kHz}$ excitation frequency.

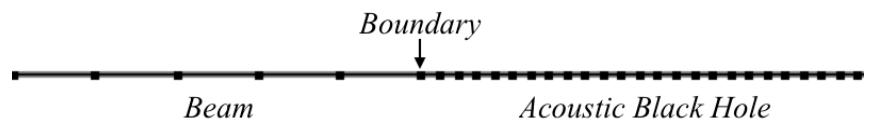

FIG. 5. The meshing difference between the beam, on the left, and the $\mathrm{ABH}$, on the right.

be used in this investigation to obtain the reflection coefficient and facilitate the following comparison of the various $\mathrm{ABH}$ configurations.

To decompose the waves in a beam, the number of sensors required is dependant on the number of waves that make up the disturbance. For example, in the case where there are both positive and negative evanescent (near-field) waves and propagating (far-field) waves, four sensors are required to form four simultaneous equations that can be used to calculate the amplitude and phase of the individual waves. However, when the sensor array is sufficiently far from any impedance changes or excitation source, the contribution from the near-field waves can be neglected and thus only two sensors are required. 
It has been assumed in the following that the near-field contribution can be neglected once it has decayed to $10 \%$ of its original magnitude, i.e.

$$
e^{-k_{f} l}=0.1
$$

where $l$ is the distance from the sensor array to any features, such as impedance changes that introduce near-field components, and $k_{f}$ is the flexural wavenumber; this is consistent with $^{28}$. To address the limits that this assumption imposes on the system geometry, it is necessary to express the flexural wavenumber in the beam as ${ }^{29}$

$$
k_{f}=\left(\frac{\rho_{s} S}{E I}\right)^{\frac{1}{4}} \omega^{\frac{1}{2}} .
$$

Substituting equation 5 into equation 4 and rearranging gives the low frequency limit as

$$
f_{\text {min }}=\frac{1}{l^{2}}\left(\frac{E I(\ln (0.1))^{4}}{4 \pi^{2} \rho_{s} S}\right)^{\frac{1}{2}}
$$

In addition, an upper frequency limit due to aliasing can be calculated based on the requirement that the distance between the two sensors must be less than half a wavelength, which from Equation 2 gives

$$
f_{\max }=\frac{1}{\Delta_{x}^{2}}\left(\frac{\pi^{2} E I}{4 \rho_{s} S}\right)^{\frac{1}{2}}
$$

In the presented study, the sensor array was located at $14 \mathrm{~cm}$ from the excitation force and $14 \mathrm{~cm}$ from the $\mathrm{ABH}$ boundary, so that $l=14 \mathrm{~cm}$ and the sensor spacing was set to $\Delta_{x}=2 \mathrm{~cm}$. These parameters give a low frequency limit of approximately $600 \mathrm{~Hz}$ and an upper frequency limit of approximately $57 \mathrm{kHz}$. However, this analysis assumes that the structure behaves as a beam with one-dimensional wave propagation, which will break down when the wavelength becomes comparable to either the width or height of the beam ${ }^{28}$. In 
the following study, this limit first occurs where the wavelength becomes comparable to the width of the beam, which is $4 \mathrm{~cm}$, and gives a practical upper frequency limit of $\sim 14 \mathrm{kHz}$. Therefore, in the following investigation the analysis has been limited to a maximum frequency of $10 \mathrm{kHz}$.

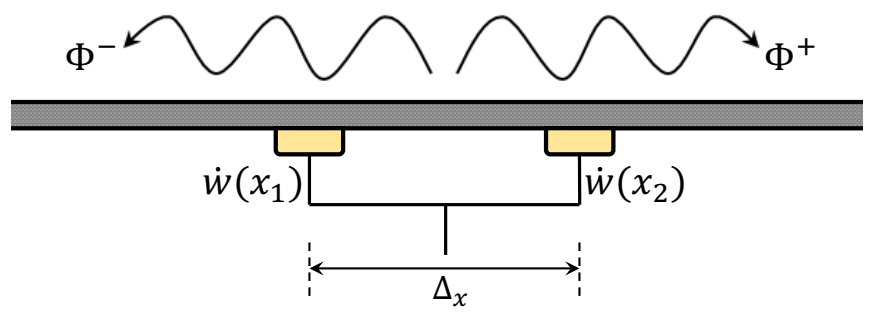

FIG. 6. Two sensors placed at $x_{1}$ and $x_{2}$ that are used to measure the velocity, $\dot{w}$, at each point. The velocity measured at each sensor is then used to calculate $\Phi^{-}$and $\Phi^{+}$, the positive and negative travelling propagating waves along the beam.

A diagram of the beam section of the model is shown in Figure 6 indicating the sensing points and the two propagating waves. Each sensor measures the velocity, which is the superposition of the two propagating waves at each point, such that

$$
\dot{w}(x)=i \omega\left(\Phi^{+} e^{-i k_{f} x}+\Phi^{-} e^{i k_{f} x}\right),
$$

where $\dot{w}$ is the transverse velocity measured at a point, $x$, along the beam and $\Phi^{+}$and $\Phi^{-}$ are the complex amplitudes of the positive and negative propagating waves respectively ${ }^{29}$. The positive and negative propagating wave amplitudes can then be calculated in terms of 
the velocity at the $n$-th sensor, $\dot{w}\left(x_{n}\right)$, as ${ }^{29}$

$$
\begin{aligned}
& \Phi^{+}=\frac{-1}{2 \omega \sin \left(k_{f} \Delta_{x}\right)}\left[\dot{w}\left(x_{1}\right) e^{\frac{i k_{f} \Delta_{x}}{2}}-\dot{w}\left(x_{2}\right) e^{\frac{-i k_{f} \Delta_{x}}{2}}\right] \\
& \Phi^{-}=\frac{-1}{2 \omega \sin \left(k_{f} \Delta_{x}\right)}\left[\dot{w}\left(x_{2}\right) e^{\frac{i k_{f} \Delta_{x}}{2}}-\dot{w}\left(x_{1}\right) e^{\frac{-i k_{f} \Delta_{x}}{2}}\right] .
\end{aligned}
$$

The magnitude of the reflection coefficient can then be calculated as

$$
|R|=\left|\frac{\Phi^{-}}{\Phi^{+}}\right| .
$$

This will be used in the following parametric study to investigate the tradeoffs in the design of an $\mathrm{ABH}$ terminating a beam.

\section{PARAMETRIC STUDY AND MODAL ANALYSIS}

In this section, the results from the parametric study of an $\mathrm{ABH}$ on a beam are presented. In particular, the effects over frequency of varying the tip height, taper length and power law are investigated, before the broadband design is considered. A parametric study was chosen for this investigation rather than a direct optimisation procedure as highlighted in ${ }^{16}$, to enable the intricate effects that each design parameter has on the reflection coefficient of the $\mathrm{ABH}$ over a broad frequency and parameter range to be examined. Although this parametric study can ultimately be used to assess optimal design parameters, it is not restricted to a specific optimisation cost function and, therefore, is able to provide broader insight. That said, it would be more appropriate to perform a direct optimisation process if an $\mathrm{ABH}$ was to be designed for a specific application and thus utilise such methods as outlined in ${ }^{16}$.

The reflection coefficient for each parameterisation has been calculated using the method defined in Section IIC over a frequency range of $100 \mathrm{~Hz}$ to $10 \mathrm{kHz}$, which is well within 
the upper and lower limits of validity, as discussed in Section IIC. In addition, for each parameterisation, the modes of the $\mathrm{ABH}$ cell have been calculated by modelling the cell in isolation and assuming a no-rotation boundary condition at the junction between the beam and the ABH. The no-rotation boundary condition was found to approximate the modal behaviour of the ABH coupled to the beam section well, because, in the fully-coupled system, the rotational stiffness of the beam at the $\mathrm{ABH}$ junction is much greater than the bending stiffness.

\section{A. The effect of the tip height}

Figure $7(\mathrm{a})$ shows a contour plot of the reflection coefficient plotted as a function of frequency for a range of tip heights from $0.01 \mathrm{~mm}$ to $3 \mathrm{~mm}$, which has been chosen to cover practically realisable tip heights. The taper length has been fixed at $70 \mathrm{~mm}$ and the power law set to $\mu=4$. The resolution of the change in the tip height was decreased iteratively until the results shown in Figure 7 (a) converged. The tip height was ultimately varied in steps of $6.67 \mu \mathrm{m}$, which corresponds to $1 / 5$ of the minimum flexural wavelength.

The results in Figure 7(a) show that as frequency increases from $100 \mathrm{~Hz}$ to $10 \mathrm{kHz}$, there are varying bands of high and low reflection coefficient. At larger tip heights, the spectral bands become wider in both bandwidth and spacing and, resultantly, there are fewer bands of low reflection within the presented frequency range. Interestingly, it is also clear that the minima in the spectral bands are lower in the mid-range of tip heights presented and, therefore, if the $\mathrm{ABH}$ was being tuned for a narrowband control problem, there may be a benefit to selecting a tip height that is greater than the minimum manufacturable limit; this 


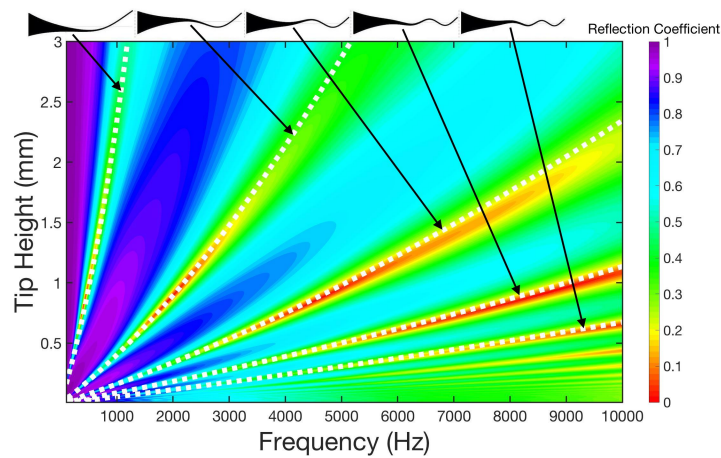

(a)

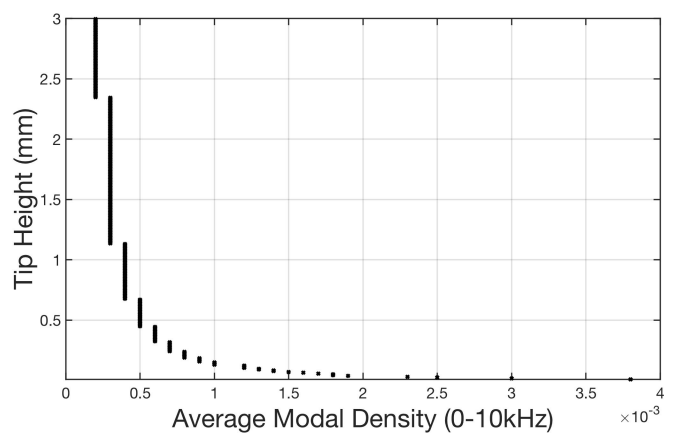

(b)

FIG. 7. (color online) (a) The reflection coefficient, shown on a colour scale of 0 to 1 , plotted as a function of frequency and tip height for an $\mathrm{ABH}$ with a taper length of $70 \mathrm{~mm}$ and a power law of 4. The modal frequencies are indicated by the white dotted lines and the first five mode shapes are shown. (b) The change in modal density averaged across the considered bandwidth as a function of the tip height.

is distinct from the general $\mathrm{ABH}$ design approach. For example, the performance at $7 \mathrm{kHz}$ can be maximised by using a tip height of $0.7 \mathrm{~mm}$. Despite this potential for narrowband tuning, by decreasing the tip height the bands of low reflection begin to overlap and the broadband performance tends to increase; this broadband performance will be explored in more detail below.

In addition to the narrowband and broadband performance of the ABHs, it is interesting to consider the low frequency performance limit. From the results presented in Figure 7(a), it can be seen that at frequencies below $\sim 2 \mathrm{kHz}$, the bands of low reflection become narrow and the performance of the $\mathrm{ABH}$ is poor for the range of tip heights examined. This low frequency performance can be related to the length of the $\mathrm{ABH}$ taper because, as noted in the introduction, an $\mathrm{ABH}$ is known to become effective when the length of the taper is 
comparable to or longer than the flexural wavelength ${ }^{2}$. Although this is somewhat difficult to measure because of the change in the wavelength over the length of the taper, an estimate can still be made by considering the wavelength halfway along the taper. For example, for a power law of $\mu=4$, a taper length of $7 \mathrm{~cm}$ and a tip height of $0.6 \mathrm{~mm}$ ), the taper becomes comparable to the wavelength at approximately $2 \mathrm{kHz}$, which generally aligns well with the results presented in Figure 7(a). However, it is clear that there is appreciable performance at lower frequencies, and that a more robust explanation for the low frequency limit can be achieved through evaluating the modes of the $\mathrm{ABH}$.

To provide further insight into the physical behaviour of the $\mathrm{ABH}$, the modes of the $\mathrm{ABH}$ cell have been calculated as described in the introduction to Section III. The variation in the frequency of the first 5 modes of the ABH cell over tip height are shown by the white dotted lines in Figure 7(a) and the corresponding mode shapes are shown for the first 5 modes. From these results it can be seen that the frequencies at which the modes occur align well with the bands of low reflection and that at low frequencies the ABH only achieves a low reflection coefficient at frequencies very close to the first mode. It can also be seen from these results that that the modal density increases with a decrease in the tip height, which is consistent with ${ }^{17}$ and Figure 7 (b) shows the change in the modal density averaged across the considered bandwidth as a function of the tip height. From this plot it can be seen that the modal density increases exponentially for a decreasing tip height. The increase in the modal density for smaller tip heights is due to higher order modes occurring at lower frequencies and this can, in turn, be related to the increased reduction in the wavelength over the length of the taper. 


\section{B. The effect of the taper length}

Figure 8(a) shows a contour plot of the reflection coefficient plotted as a function of frequency for a range of taper lengths from $10 \mathrm{~mm}$ to $300 \mathrm{~mm}$, which has been chosen to represent practically realisable taper lengths. The tip height has been fixed at $0.6 \mathrm{~mm}$ and the power law set to $\mu=4$. As in the previous section, the resolution of the change in the taper length was decreased iteratively until the results shown in Figure 8(a) converged. The taper length was ultimately varied in steps of $2 \mathrm{~mm}$, which corresponds to $1 / 10$ of the minimum flexural wavelength. The results in Figure 8(a) show that, as frequency increases, the reflection coefficient of the ABH varies in bands, similarly to Figure 7(a). For longer tapers, there are more bands of high and low reflection than for shorter tapers over the same bandwidth. As discussed in Section III A, the low frequency limit of the ABH is dependent on the length of the taper and it can be seen from the results in Figure 8(a) that the low frequency limit decreases as the taper length is increased. For the considered power law ( $\mu$ $=4)$ and tip height $\left(h_{t i p}=0.6 \mathrm{~mm}\right)$, when the taper is shorter than $2.6 \mathrm{~cm}$, the broadband performance of the $\mathrm{ABH}$ is limited over the presented frequency range. As in Section III A, this can be related to the length of the $\mathrm{ABH}$ and a $2.6 \mathrm{~cm}$ taper becomes comparable to the flexural wavelength at a frequency of $10 \mathrm{kHz}$. At taper lengths below $2.6 \mathrm{~cm}$ it can be seen from Figure 8(a) that a dip in the reflection coefficient only occurs over a narrow bandwidth around the first $\mathrm{ABH}$ mode, as discussed in the previous section. That said, considering the relatively small amount of damping assumed in the presented results, it can be seen that ABHs with longer tapers are very effective, especially at higher frequencies, where 


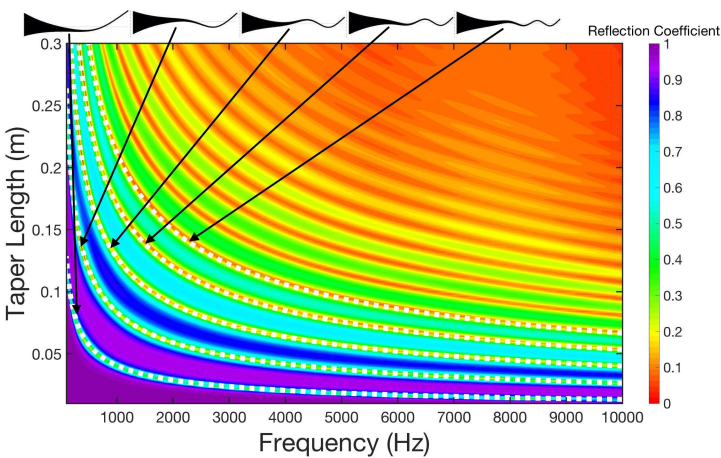

(a) bandwidth.

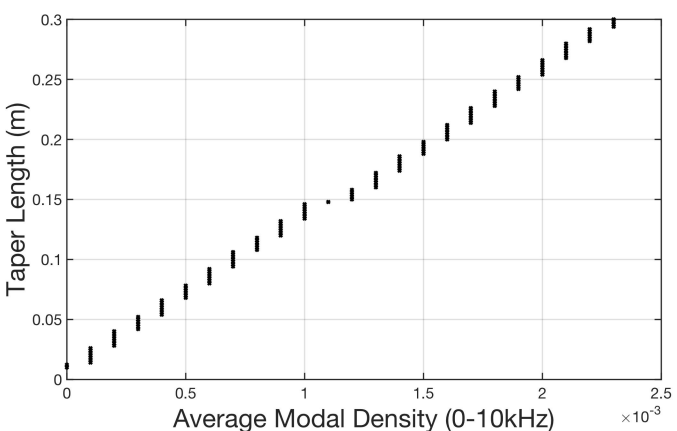

(b)

FIG. 8. (color online) (a) The reflection coefficient, shown on a colour scale of 0 to 1 , plotted as a function of frequency and taper length for an $\mathrm{ABH}$ with a tip height of $0.6 \mathrm{~mm}$ and a power law of 4. The modal frequencies are indicated by the white dotted lines and the first five mode shapes are shown. (b) The change in modal density averaged across the considered bandwidth as a function of the taper length.

the reflection coefficient is between around 0 and 0.2 over a large portion of the presented 


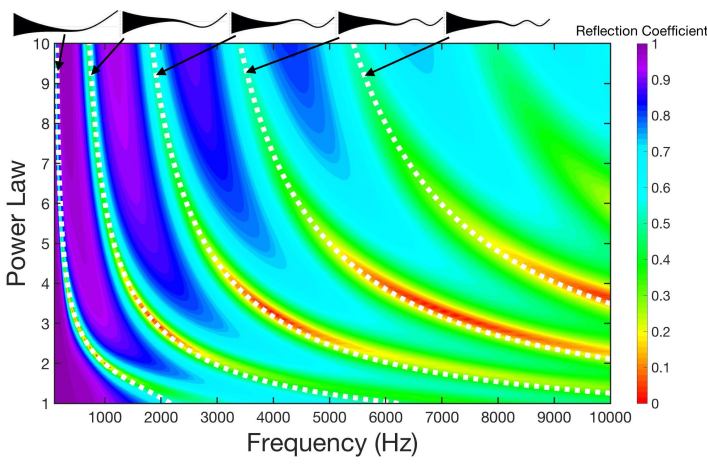

(a)

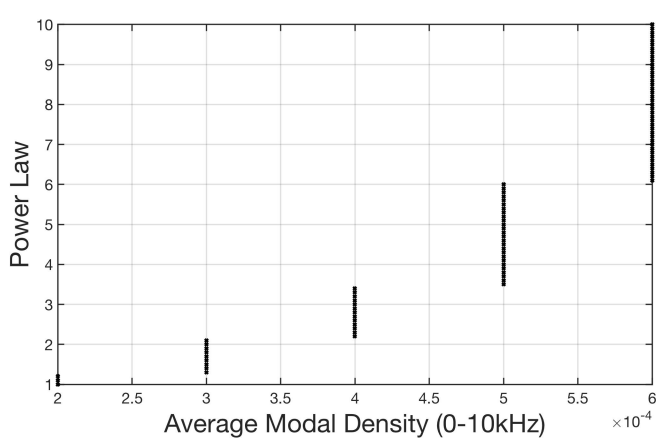

(b)

FIG. 9. (color online) (a) The reflection coefficient, shown on a colour scale of 0 to 1 , plotted as a function of frequency and power law for an $\mathrm{ABH}$ with a tip height of $0.6 \mathrm{~mm}$ and a taper length of $70 \mathrm{~mm}$. The modal frequencies are indicated by the white dotted lines and the first five mode shapes are shown. (b) The change in modal density averaged across the considered bandwidth as a function of the power law. 
From the results presented in Figure 9(a) it can be seen that the spectral bands of high and low reflection, observed previously, also vary with the taper power law. At higher frequencies, on average, the reflection coefficient is lower for all power laws and this is consistent with the previous results and literature. Figure 9(a) once again shows the alignment between the modal frequencies of the $\mathrm{ABH}$ and the bands of low reflection for the range of power laws examined. A higher power law changes the mass distribution of the taper and can be seen to lower the modal frequencies and thus increase the modal density over the presented frequency range. This is also shown by the average modal density versus power law, which is shown in Figure 9(b). From this plot it can be seen that the modal density increases exponentially with the power law. In addition to the changes in the modal frequencies, it can be seen from the results shown in Figure 9(a) that for power laws less than around 5, the minima in the spectral bands are lower than for higher power laws. For example, in the band corresponding to the third mode, the reflection coefficient is lowest for a power law between 3 and 5 . There thus exists an optimal power law, which reaches a tradeoff between the large impedance change between the beam and the taper at higher power laws, and the limited length of the taper over which the wave speed is relatively slow at lower power laws. That is, the reflection at higher power laws becomes dominated by the component reflected from the junction to the $\mathrm{ABH}$ rather than from the $\mathrm{ABH}$ itself, as demonstrated in ${ }^{18}$, and it can be seen from the results in Figure 9(a) that this is a frequency dependent effect.

Based on the above discussion and the results in Figure 9(a), it is evident that there is an optimum power law that can be used to attenuate a particular frequency. For example, if attenuation is required at $7 \mathrm{kHz}$ and the $\mathrm{ABH}$ has been constrained to a length of $7 \mathrm{~cm}$ and 
a tip height of $0.6 \mathrm{~mm}$, the optimum power law would be 3.3. This introduces the idea that

the power law can be used to tune the behaviour of an $\mathrm{ABH}$ when the other key geometrical parameters, namely the length and tip height, are constrained due to practical restrictions.

\section{Parameter selection for optimal design}

It has been shown in the previous sections that the geometrical parameters of an $\mathrm{ABH}$ can be tuned to achieve a change in its performance characteristics. In particular, it has been discussed how the parameters can be tuned to optimise the ABH for performance at a single frequency or over a narrowband. However, the ABH design parameters could also be tuned to minimise the reflection over a broadband frequency range and in this case the optimal parameters will depend on both the bandwidth of interest and the constraints due to the application. In this section, the potential design tradeoffs will be considered for the case when the maximum broadband performance of the $\mathrm{ABH}$ is required and the optimal design parameters will be evaluated. In this investigation, the broadband performance will be assessed over a frequency range of $100 \mathrm{~Hz}-10 \mathrm{kHz}$ by calculating the average reflection coefficient, and the minimum broadband reflection coefficient over the parameter space will be evaluated.

In the first instance, Figure 10 shows how the broadband average reflection coefficient varies with both taper length and tip height for an $\mathrm{ABH}$ with a power law of 4 . From these results it can be seen that increasing the length of the taper lowers the broadband average reflection coefficient for this power law. The optimal configuration is thus relatively trivial in this case, essentially requiring the longest taper length achievable. That said, for each 
taper length there is an optimum tip height and it is, therefore, insightful to discuss the behaviour further.

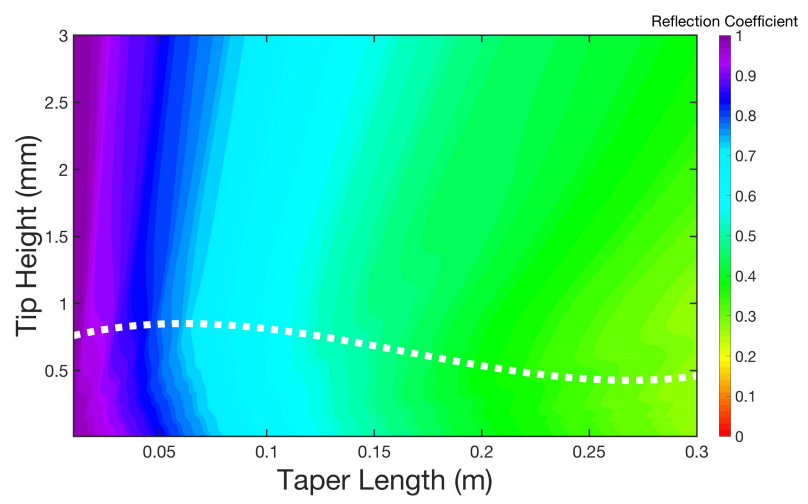

FIG. 10. (color online) The variation in the broadband average reflection coefficient with both the tip height and taper length of an $\mathrm{ABH}$ with a power law of 4. The reflection coefficient has been averaged over a broadband frequency range $(100 \mathrm{~Hz}-10 \mathrm{kHz})$ and is shown on a colour scale of 0 to 1 . The optimum tip height is shown by the dotted white line.

For the parameter ranges examined, it is clear that the optimum tip height, which minimises the broadband reflection coefficient for a specific taper length, varies with taper length. This is shown by the dotted white line in Figure 10 and is generally lower for longer taper lengths. This is somewhat distinct from current ABH design strategies, which specify that reducing the tip height improves the performance of the $\mathrm{ABH}$. This is because, as shown in Section III A for a power law of 4, that although a small tip height gives a higher modal density, the minima in the bands of low reflection are low enough to shift the minimum in the broadband average up to the mid-range tip heights. This may explain the lower optimum tip height at longer taper lengths because, as shown in Section III B, increasing the taper length reduces the reflection coefficient at all frequencies and would therefore reduce 
the difference between the minima in the bands of low reflection in Section III A and the reflection coefficient when the modal density is high. It should be noted then that the optimum tip height for minimising the broadband reflection coefficient may not be suitable for all broadband vibrational problems as there may be cases where the problematic frequencies do not align with the bands of low reflection.

Figure 11 shows how the broadband average reflection coefficient varies with both the power law of the $\mathrm{ABH}$ and the length of the taper. For a fixed power law, the results show that increasing the length of the taper decreases the broadband average reflection coefficient. As shown in Section III B, increasing the length of the taper increases the modal density and, therefore, increases the attenuation provided by the ABH. A more interesting observation from the results presented in Figure 11 is, however, that at each taper length there is an optimum power law that can be used to achieve the lowest broadband reflection and this power law has been indicated by the dotted white line.

From the indicated optimal results shown in Figure 11, it can be seen that the optimum power law varies with taper length. In section IIIC, it was shown that increasing the power law results in an increase in the modal density, but also increases the reflection from the junction between the beam and the ABH. This trade-off differs for each taper length, due to the corresponding variation in the modal density as discussed in Section III B. Specifically, for a long taper with a high modal density, a lower power law is used to limit the impedance change and, therefore, reflection at the junction. Whereas for a shorter taper, with a relatively low modal density, a higher power law provides the optimal trade-off between reflection from the junction and modal density. This trend can be seen from the 


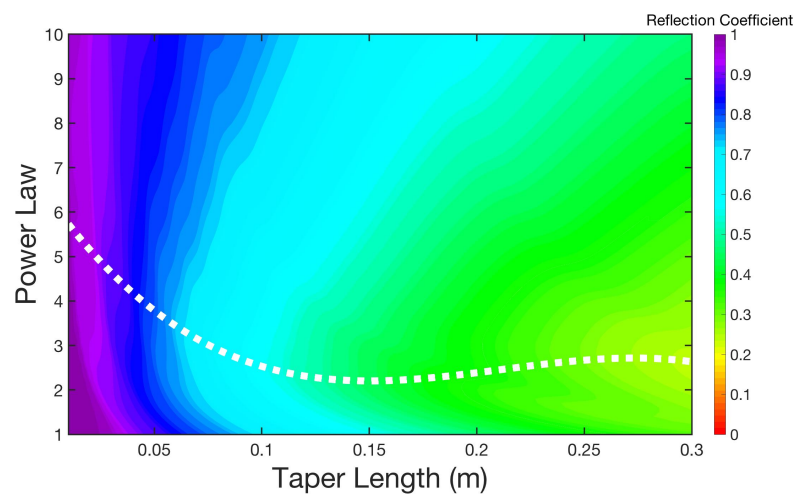

FIG. 11. (color online) The variation in the broadband average reflection coefficient with both the power law of the taper and the taper length for an ABH with a tip height of $0.6 \mathrm{~mm}$. The broadband reflection coefficient is shown on a colour scale from 0 to 1 . The optimum power law is shown by the dotted white line.

results presented in Figure 11. It is also interesting to note that for taper lengths greater than about $10 \mathrm{~cm}$, the optimum power law is relatively constant with a value between 2 and 3. In summary, the results in Figure 11 show that the power law of an $\mathrm{ABH}$ can be optimised for a specific taper length to achieve the minimum broadband reflection coefficient. For example, if the length of the ABH taper was constrained by the intended application, the power law of the ABH could be optimally tuned according to the data shown in Figure 11.

In addition to considering how the broadband performance varies with both power law and taper length, it is interesting to consider the variation with power law and tip height and this is shown in Figure 12. The optimum power law, which minimises the broadband reflection coefficient for each tip height, is shown by the dotted white line. From these results it can be seen that the optimum power law is greater for larger tip heights and this can be 
417

422

423

related to a shift in the trade-off between modal density and reflection from the junction. That is, when the modal density is limited by the tip height, the benefit of increasing the modal density by using a higher power law outweighs the relative change in the reflection from the junction to the ABH. For smaller tip heights, this balance between the reflection from the junction and the modal density occurs at a lower power law.

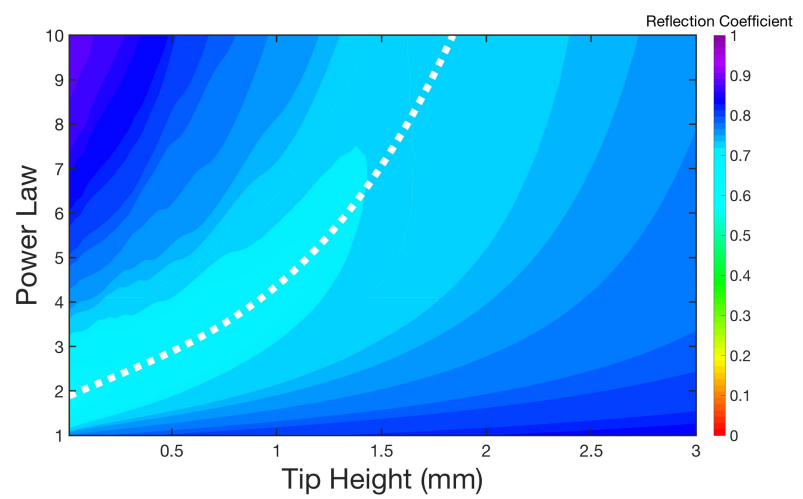

FIG. 12. (color online) The variation in the broadband average reflection coefficient with both the power law of the taper and the tip height of an $\mathrm{ABH}$ with a taper length of $70 \mathrm{~mm}$. The broadband reflection coefficient is shown on a colour scale from 0 to 1 . The optimum power law is shown by a dotted white line.

\section{EXPERIMENTAL VALIDATION}

In this section, the reflection coefficient of a practical $\mathrm{ABH}$ will be presented and the experimentally identified modes will be assessed to validate the physical insights provided by the simulation-based study. This experimental study will demonstrate that the practical $\mathrm{ABH}$ behaves with the characteristics that were predicted in the simulation study. 


\section{A. Experimental setup}

An $\mathrm{ABH}$ on a beam was cut from an aluminium plate, alloy 8082-T6, using a water jet and the dimensions are shown in Table II. The manufactured beam with the ABH termination is shown in Figure 13, with and without the additional damping applied to the taper. The damping that was used in this experiment was 'yellow plastic compound', manufactured by WT Henley ${ }^{30}$. The compound is easy to mould and was stuck to the aluminium surface without requiring additional adhesive. The structure was then mounted, via a force gauge, onto a large shaker as shown in Figure 14. The shaker was driven with white noise, using a sample time of $41.7 \mu$ s (corresponding to a Nyquist frequency of $12 \mathrm{kHz}$ ).

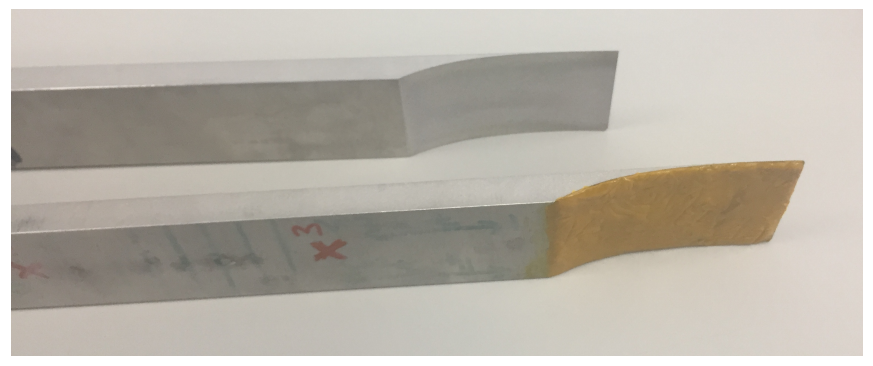

FIG. 13. (color online) A picture of the ABH that was used in the experimental study, with and without damping.

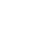

The resulting vibration of the structure was measured at intervals of $5 \mathrm{~mm}$ along the length of the beam and the taper sections using a Polytec PDV-100 laser vibrometer mounted on a tripod $210 \mathrm{~mm}$ above the ABH. Each measurement was taken for a duration of $60 \mathrm{~s}$ to allow significant averaging to achieve good coherence. The reflection coefficient was then calculated using the wave decomposition method, described in section II C. A good signal to noise ratio was observed by using a sensor separation of $2 \mathrm{~cm}$. Based on the 
TABLE II. The dimensions of the manufactured ABH on a beam.

\begin{tabular}{cc}
\hline \hline Parameter & Value \\
\hline Beam height & $10 \mathrm{~mm}$ \\
Beam length & $300 \mathrm{~mm}$ \\
Beam width & $40 \mathrm{~mm}$ \\
ABH tip height & $0.5 \mathrm{~mm}$ \\
ABH taper length & $70 \mathrm{~mm}$ \\
ABH width & $40 \mathrm{~mm}$ \\
ABH power law & \\
Damping layer thickness & 4 \\
\hline
\end{tabular}

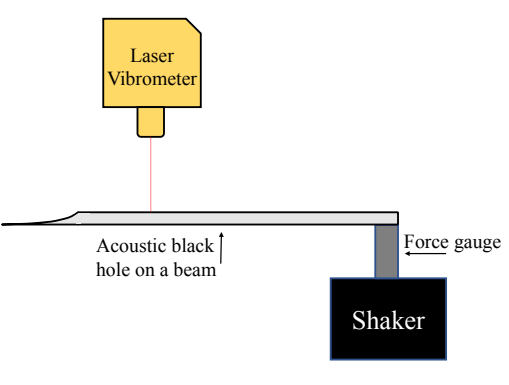

$\mathrm{a}$

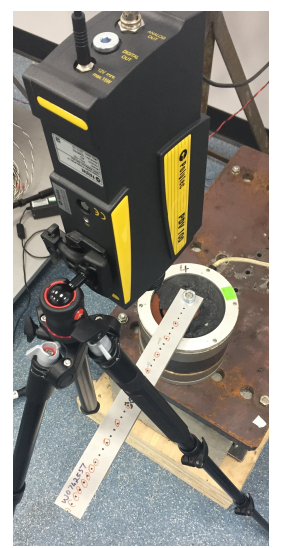

b

FIG. 14. (color online) A diagram (a) and picture (b) of the experimental setup used. 
experimental dimensions, the lower frequency measurement limit was $\sim 600 \mathrm{~Hz}$ (based on the distance between the sensor and any near field components) and the upper frequency measurement limit was $\sim 14 \mathrm{kHz}$ (based on the assumption that there are no flexural modes across the width or height of the beam).

\section{B. Results}

Figure 15 shows the measured reflection coefficient over frequency along with the measured mode shapes of the ABH cell and their frequencies. The small peaks in the data, such as at approximately $4.3 \mathrm{kHz}$, occur at frequencies where the coherence of the measured signal was limited due to the location of the measurements with respect to nodal points on the beam. Minima in the reflection coefficient occur at $1.3 \mathrm{kHz}, 3.35 \mathrm{kHz}, 6.01 \mathrm{kHz}$ and $9.45 \mathrm{kHz}$. The modes of the ABH cell were extracted by examining the amplitude of the displacement along the taper and have been numbered in Figure 15. The first five modal frequencies match the frequencies of the bands of low reflection, with the first mode occurring at approximately $315 \mathrm{~Hz}$. The first mode falls outside of the valid frequency range of the wave decomposition and therefore the reflection coefficient measured at this frequency is not valid, but is still included for reference. Figure 15 also shows finite element results for the same ABH design parameters, where the mass and loss factor of the yellow damping material have been matched in the model as discussed in Section II A. Although there are slight deviations, the finite element results match the experimental results well and validate the insight gained from the model based investigation. 


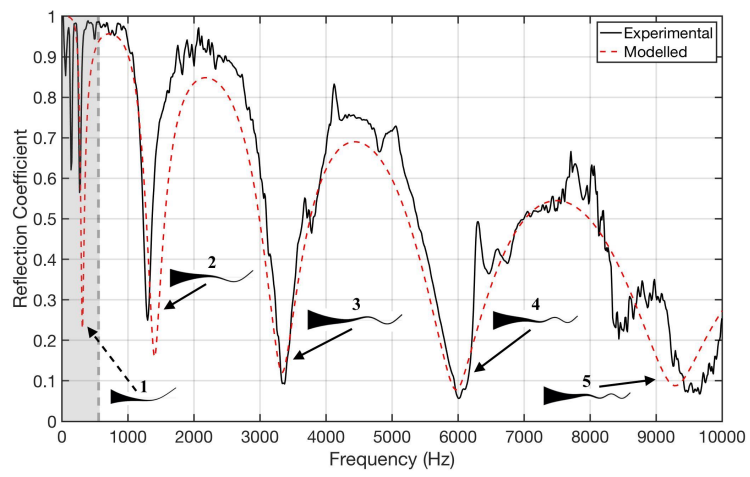

FIG. 15. The reflection coefficient calculated using the experimentally measured velocities from an $\mathrm{ABH}$ on a $300 \mathrm{~mm}$ beam. The dimensions of the beam and $\mathrm{ABH}$ are specified in Table II and the measured mode shapes at each minima have been plotted. The grey shaded area is the frequency range that the wave decomposition is invalid for. In addition, the reflection coefficient calculated using the FE model is shown.

\section{CONCLUSIONS}

This article has presented an extended study of how the controllable geometrical parameters of an $\mathrm{ABH}$ influence the reflection coefficient and the broadband average reflection coefficient of a beam. A finite element model has been developed and utilised to carry out a parametric design study. In the first instance, the variation in the reflection coefficient over frequency has been investigated as either the tip height, taper length or power law of the ABH are modified. These results have shown that the reflection coefficient exhibits bands of low reflection and, through a modal analysis, these bands have been linked to the modes of the ABH cell. As a result of this insight, it has been shown how the ABH can be tuned for optimal performance at either a single frequency or in a broadband sense. In the case of the 
tip height, it has been shown that although a smaller tip height increases the modal density, greater levels of narrowband attenuation can be achieved with an increased tip height. An optimal tip height is, therefore, shown to exist. It has also been shown that the modal density and the performance of the $\mathrm{ABH}$ is increased by increasing the taper length. In terms of the power law, it has been shown that a higher power increases the modal density, but this does not necessarily minimise the reflection coefficient. It is shown that there is an optimal setting for the power law that must reach a trade-off between maximising the modal density and limiting the reflection from the junction between the uniform beam and the taper, which becomes significant at higher power laws.

Although the presented narrowband results are consistent with individual parameterisations already presented in the literature, the presented parameter sweep over a broadband frequency range provides clear insight into the tuning of an $\mathrm{ABH}$ under particular design constraints. It has also provided new physical insight into the trade-off that must be considered when selecting the power law, as previously discussed in ${ }^{15}$, but not directly linked to the underlying physical behaviour and the tip height. That is, it is highlighted through the presented study that the trade-off is between maximising the modal density, which occurs for higher power laws and smaller tip heights, and limiting the reflection from the junction to the $\mathrm{ABH}$.

The broadband parameter sweep has also fed into an investigation into the variation in the broadband averaged reflection coefficient with the three geometrical design parameters. This has initially shown that the broadband average reflection coefficient is minimised by maximising the taper length as expected. However, contrary to current ABH design strate- 
gies, the tip height was shown to have an optimum value for a specific $\mathrm{ABH}$ parameterisation. In practical applications, the tip height and taper length are likely to be constrained and, therefore, a series of results have also been presented that demonstrate how the power law should be optimally tuned depending on other design constraints. In overview, it has been shown that the optimal power law decreases with the taper length and increases with the tip height and this observation has been linked to reaching a trade-off between maximising the modal density and limiting the reflection from the junction between the beam and the taper.

Finally, an experimental case study has been presented that validates the $\mathrm{ABH}$ behavioural trends predicted by the parametric numerical study. That is, the bands of low reflection were shown to align with the experimentally identified ABH modes, thus supporting the presented numerical analysis.

\section{ACKNOWLEDGMENTS}

This work was supported by an EPSRC iCASE studentship (Voucher number 16000058) and an EPSRC Prosperity Partnership (EP/S03661X/1).

The authors acknowledge the use of the IRIDIS High Performance Computing Facility, and associated support services at the University of Southampton, in the completion of this work. 


\section{REFERENCES}

${ }^{1}$ Krylov, V.V. and Tilman, F. (2004). "Acoustic 'black holes' for flexural waves as effective vibration dampers," Journal of Sound and Vibration 274, 605-619.

${ }^{2}$ Conlon, S.C. and Feurtado, P.A. (2014). "Progressive phase trends in plates with embedded acoustic black holes," The Journal of the Acoustical Society of America 143(2), $921-930$.

${ }^{3}$ Mironov, M. (1988). "Propagation of a flexural wave in a plate whose thickness decreases smoothly to zero in a finite interval," Soviet Physics: Acoustics 34(3), 318-319.

${ }^{4}$ Bowyer, E.P., OBoy, D.J., Krylov, V.V. and Horner, J.L. (2012). "Effect of geometrical and material imperfections on damping flexural vibrations in plates with attached wedges of power law profile," Applied Acoustics 73, 514-523.

${ }^{5}$ Huang, W., Ji, H., Qiu, J. and Cheng, L. (2016). "Wave Energy Focalization in a Plate With Imperfect Two-Dimensional Acoustic Black Hole Indentation," Journal of Vibration and Acoustics 138, 1-12.

${ }^{6}$ Denis, V., Pelat, A. and Gautier, F. (2016). "Scattering effects induced by imperfections on an acoustic black hole placed at a structural waveguide termination," Journal of Sound and Vibration 362, 56-71.

${ }^{7}$ Ji, H., Luo, J., Qiu, J., and Cheng, L. (2018). "Investigations on flexural wave propagation and attenuation in a modified one-dimensional ABH using a laser excitation technique," Mechanical systems and signal processing 104, 19-35. 
${ }^{8}$ Zhao, L. (2016). "Passive vibration control based on embedded acoustic black holes," Journal of Vibration and Acoustics 138, 1-6.

${ }^{9}$ Bowyer, E.P. and Krylov, V.V. (2014). "Experimental investigation of damping flexural vibrations in glass fibre composite plates containing one- and two-dimensional acoustic black holes," Composite Structures 107, 406-415.

${ }^{10}$ Lee, J.Y. and Jeon, W. (2016). "An optimal design of 1D Acoustic Black Hole with damping layer for plate geometry," (Proceedings of the 23rd International Congress of Sound and Vibration), 10-14.

${ }^{11}$ Feurtado, P.A. and Conlon, S.C. (2016). "An experimental investigation of acoustic black hole dynamics at low, mid, and high frequencies," Journal of Vibration and Acoustics 138, $1-6$.

${ }^{12}$ Georgiev, V.B., Cuenca, J., Gautier, F., Simon, L. and Krylov, V.V. (2011). "Damping of structural vibrations in beams and elliptical plates using the acoustic black hole effect," Journal of Sound and Vibration 330(11), 2497-2508.

${ }^{13}$ Kerwin Jr., E.M. (1959). "Damping of flexural waves by a constrained viscoelastic layer," The Journal of the Acoustical Society of America 31(7), 952-962.

${ }^{14}$ Feurtado, P.A. and Conlon, S.C. (2014). "A normalized wave number variation parameter for acoustic black hole design," The Journal of the Acoustical Society of America 136(2), $148-152$.

${ }^{15}$ Shepherd, M.R., Feurtado, P.A. and Conlon, S.C., (2016). "Multi-objective optimization of ABH vibration absorbers," The Journal of the Acoustical Society of America 140(3), 
$227-230$

${ }^{16}$ McCormick, C.A. and Shepherd, M.R. (2018). "Optimal design and position of an embedded one-dimensional acoustic black hole," (Proceedings of Inter-Noise 2018, Chicago, IL, USA), 26-29.

${ }^{17}$ Denis, V., Pelat, A., Gautier, F. and Elie, B. (2014). "Modal overlap factor of a beam with an ABH termination," Journal of Sound and Vibration 333(12), 2475-2488.

${ }^{18}$ Karlos, A., Elliott, S. and Cheer, J. (2019). "Higher-order WKB analysis of reflection from tapered elastic wedges," Journal of Sound and Vibration 449, 368-388.

${ }^{19}$ O’Boy, D.J., Krylov, V.V., Kralovic, V. (2010). "Damping of flexural vibrations in rectangular plates using the acoustic black hole effect," Journal of Sound and Vibration 329(22), 4672-4688.

${ }^{20}$ Tang, L., Cheng, L., Ji, H., and Qiu, J. (2016). "Characterization of acoustic black hole effect using a one-dimensional fully-coupled and wavelet-decomposed semi-analytical model," Journal of Sound and Vibration 374, 172-184

${ }^{21}$ Feurtado, P.A. and Conlon, S.C. (2015). "Investigation of boundary-taper reflection for acoustic black hole design," Journal of Noise Control Engineering 5, 460-466.

${ }^{22}$ Pelat, A., Denis, V. and Gautier, F. (2015). "Experimental and theoretical study of the reflection coefficient of an ABH beam termination," (INTER-NOISE and NOISE-CON Congress and Conference Proceedings) 250 (2), 5001-5009.

${ }^{23}$ Conlon, S.C., Fahnline, J.B. and Semperlotti, F. (2015). "Numerical analysis of the vibroacoustic properties of plates with embedded grids of acoustic black holes," Journal of 
the Acoustical Society of America 137(1), 447-457.

${ }^{24}$ Marburg, S. (2008). "Discretization requirements: How many elements per wavelength are necessary?," in Computational Acoustics of Noise Propagation in Fluids - Finite and Boundary Element Methods, (Springer).

${ }^{25}$ Song, B.H. and Bolton, J.S. (2000). "A transfer-matrix approach for estimating the characteristic impedance and wave numbers of limp and rigid porous materials," The Journal of the Acoustical Society of America 107(3), 1131-1152.

${ }^{26}$ Halkyard, C. and Mace, B. (1995). "Structural intensity in beams-waves, transducer systems and the conditioning problem," Journal of Sound and Vibration 185(2), 279-298.

${ }^{27}$ Halkyard, C. and Mace, B. (2002). "Feedforward adaptive control of flexural vibration in a beam using wave amplitudes," Journal of Sound and Vibration 254(1), 117-141.

${ }^{28}$ Denis, V., Gautier, F., Pelat, A. and Poittevin, J. (2015). "Measurement and modelling of the reflection coefficient of an ABH termination," Journal of Sound and Vibration 349, $67-79$

${ }^{29}$ Fuller, C.R., Elliott, S.J., and Nelson, P.A. (1996). Active Control of Vibration (Academic Press).

${ }^{30}$ Henley, W.T. (2018). "Yellow plastic compound," https://www.wt-henley •com/cable_ accessories-green_and_yellow_plastic_compound.html. 